\title{
ARE CONTRAST SENSITIVITY FUNCTIONS IMPAIRED IN INSULIN DEPENDENT DIABETICS WITHOUT DIABETIC RETINOPATHY?
}

\author{
Vladimír Liška, Miroslav Dostálek
}

City Hospital, Litomyšl: Department of Ophthalmology

\begin{abstract}
Summary: Purpose: To confirm the influence of multilevel metabolic disturbance of insulin dependent diabetes mellitus (IDDM) on the vision even before the onset of the other changes routinely evaluated by ophthalmologists. Methods: Contrast sensitivity functions (CSFs) were estimated using the VCTS 6500 board. The standardised measurement procedure was performed. The value of the threshold contrast sensitivity was obtained for five spatial frequencies (1.5 - 3 - 6 - 12 - $18 \mathrm{c} / \mathrm{deg}$ ). Other data was collected (duration of diabetes, BCVA, funduscopy, fluoresceine angiography, HbA1C). The study group consisted of 48 IDDM patients (94 eyes) without diabetic retinopathy and with Snellen BCVA > 1.0. The control group (56 normals, 98 eyes) was age and BCVA matched. Results: Highly statistically significant decrease of the CSFs in all spatial frequencies in the study group was obtained. Correlation between duration of the diabetes and impaired degree of CSFs was present in the middle spatial frequency. No significant changes in CSFs were found among patients with pathological value of glycated hemoglobin HbA1c (> 7,8 \%). Conclusions: If compared with routinely used Snellen visual acuity, the CSFs are more complex descriptors of the subjects vision abilities. IDDM has an influence on these sensitive functions, especially during examination in the middle spatial frequency of 6 and $12 \mathrm{c} / \mathrm{deg}$, before disturbing visual acuity and before changes in the retinal morphology. Decrease of CSFs was influenced mainly by the patients' age and partially (in the middle spatial frequency) by the IDDM duration.
\end{abstract}

Key words: Contrast sensitivity function (CSF); Insulin dependent diabetes mellitus (IDDM); Glycated hemoglobin (HbAlc)

This paper has been presented at the Annual conference of the Czech Ophthalmological Society 1998, Hradec Králové, Czech Republic and as a poster at the XXVIIIth International Congress of Ophthalmology 1998, Amsterdam, The Netherlands.

\section{Introduction}

By means of contrast sensitivity examination we can obtain information concerning differentiation abilities of the eye on larger retina's surface and with submaximal contrasts of outer environment. The central visual acuity tested on Snellen's optotypes by maximal contrast is just one of the points on contrast sensitivity functions (CSFs) curve.

This paper focuses on confirming or disproving theories which suggest, that during longterm insulin dependent diabetes (IDDM), gradual impairment of the retina occurs, even earlier than appearance of diabetic retinopathy symptoms. In order to this we study the relationship between the length of diabetes' duration and its longterm metabolic control determined by glycated hemoglobin (HbA1c) level as well as the contribution of the patient's age.

\section{Methods and material}

All diabetics as well as health volunteers underwent a comprehensive ophthalmologic evaluation completed by estimation of the contrast sensitivity functions (CSFs).

The set of measured data was recorded in the following structure:

- Personal and anamnestic data.

- Objective refraction: measured by automatic keratorefractometer Luneau L-60 (Chartres, France).

- Subjective visual acuity: by means of the standard Snellen's optotypes, uncorrected (UCVA) or with the best subjective correction (BCVA).

- Biomicroscopy of anterior segment and fundus (both eyes).

- Photo of fundus (both eyes) and fluoresceine angiography (both eyes) recorded by funduscamera Canon 60-UV (Kawasaki, Japan). 
- Testing of glycaemia and glycated haemoglobin HbA1c: quantitative measurement in anticoagulated whole blood by the Abbott IMx Glycated Hemoglobin Assay (Abbott Laboratories, Abbott Park, IL., USA).

- Contrast sensitivity functions (CSFs): static method by means of contrast board VCTS 6500 (Vistech Consultants, Inc., Dayton, Ohio, USA) from 3 meters, by spatial frequencies 1,5-3,0-6,0-12-18 c/deg, by standard illumination $110-240 \mathrm{~cd} / \mathrm{m}^{2}$ measured with luxmeter delivered with board (Vistech Consultants, Inc., Dayton, Ohio, USA).

VCTS 6500 board is intended for examination from 3 meters. The five rows of the circular targets present space frequencies 1,5-3-6-12-18 cycles per degree (c/deg), where contrast goes down from left to right, while spatial frequency is constant. The board was read monocularly as a text's page, from left to right and from up to down. Towards the end of each row of targets, if the subject stated they could not read the grating certainly, they were asked to guess. The last correct response was considered the threshold. Regarding the fact that the tested persons had three answer possibilities at their disposal (left, right and up), in this way they underwent a three-alternative test of forced choice. The non-paired Student t-test was used for statistical analysis. All experiments were done in accord with the Helsinki Declaration.

We tested 94 eyes of 48 persons with IDDM with BCVA equal or better 1.00 , without symptoms of diabetic retinopathy, who were divided into two age groups according to the biological age. Both of these groups were divided to subgroups according to the duration of the diabetes and according to the HbAlc level:

A) age under 35 (37 eyes of 19 persons, average age 27,9 \pm 6.8)

- diabetes duration less than 10 years (27 eyes of 14 persons, average age $28.9 \pm 5.8$ )

- diabetes duration more than 10 years ( 10 eyes of 5 persons, average age $26.2 \pm 8.5$ )

- HbA1c level less 7.8\% (13 eyes of 7 persons, average age $28.4 \pm 6.3$ )

- HbA1c level more $7.8 \%$ (24 eyes of 12 persons, average age $26.9 \pm 7.8$ )

B) age 35 and over (57 eyes of 29 persons, average age 49,3 14.3)

- diabetes duration less than 10 years (38 eyes of 18 persons, average age $8.6 \pm 15.0$ )

- diabetes duration more than 10 years ( 19 eyes of 10 persons, average age $50.1 \pm 13.5)$

- HbA1c level less $7.8 \%$ (25 eyes of 13 persons, average age $47.9 \pm 15.7)$

- HbA1c level more 7.8\% (32 eyes of 17 persons, average age $49.9 \pm 13.5)$

Analogously, we tested 98 eyes of 56 healthy persons who did not suffer any eye or general disease. Also this group was divided accordingly to their age:

A) age under 35 (38 eyes of 21 persons, average age 27,6 \pm 7.2).
B) age 35 and over (60 eyes of 35 persons, average age 48,9 \pm 13.9).

The information about the significance and the design of the study was offered. Informed consent was obtained prior to participation in the study.

\section{Results}

The contrast sensitivity functions (CSFs) measured in healthy volunteers over 35 years differs only partially from curves collected from younger healthy persons. Marginally statistically significant differences were recorded only in the spatial frequencies of $6 \mathrm{c} / \mathrm{deg}$ and $18 \mathrm{c} / \mathrm{deg}$ (see Fig. 1a). Numeric values of parameter $p$ are noted in the footnotes of the particular figure, individually for each tested spatial frequency. On the contrary, differences between CSFs curves obtained by measurement in diabetics over 35 years and in diabetics under 35 years was significantly different in all spatial frequencies measured (see Fig. 1b). In both groups, however, we could find the same tendency to decrease of CSFs in subjects over 35 years.

Figs. $2 \mathrm{a}$ and $2 \mathrm{~b}$ represent cross-sectional comparisons of the data summarised on figures $1 \mathrm{a}$ and $1 \mathrm{~b}$. A statistically significant decrease of the CSFs in younger (age $<35$ years) and older (age $>35$ years) diabetics if compared to age - matched controls were revealed in all measured spatial frequencies. The differences of the CSFs in older group are more pronounced.

To investigate the relation between the decrease of CSFs and duration of IDDM, we have compared the CSFs measurements recorded in patients with history od disease shorter than 10 years with the data received from patients with longer disease duration. A statistically significant decrease of the CSFs in patients with longer IDDM anamnesis (> 10 years) was revealed in the group of younger patients (age $<35$ years) in spatial frequencies of $1.5 \mathrm{c} / \mathrm{deg}$, $6 \mathrm{c} / \mathrm{deg}$ and $12 \mathrm{c} / \mathrm{deg}$ and in the group of older diabetics (age $>35$ years) in spatial frequencies of $6 \mathrm{c} / \mathrm{deg}$. Other obtained data statistically differ insignificantly (see Fig. 3a and Fig. 3b).

The last, cross-sectional, analysis was performed to elucidate the relationship between the long-lasting metabolic compensation of the IDDM disease, the age of patients and CSFs. The level of glycated hemoglobin $\mathrm{HbAlc}=7.8 \%$ was used as a borderline for good, long-lasting metabolic compensation of the diabetes. Generally, no statistically significant diferences were revealed by comparing of the CSFs measurements in IDDM patients with good and non-sufficient metabolic control. This observation was similar in the group of younger (age $<35$ years) and older (age $>35$ years) diabetics (see. Fig. 4a and Fig. 4b). The only recorded statistically significant difference was in the subgroup of younger diabetics (age $<35$ years). On the spatial frequency of $12 \mathrm{c} / \mathrm{deg}$ a significant decrease of CSFs was recorded in the subgroup of poorly metabolically compensated diabetics $(\mathrm{HbAlc}>7.8 \%)$. 


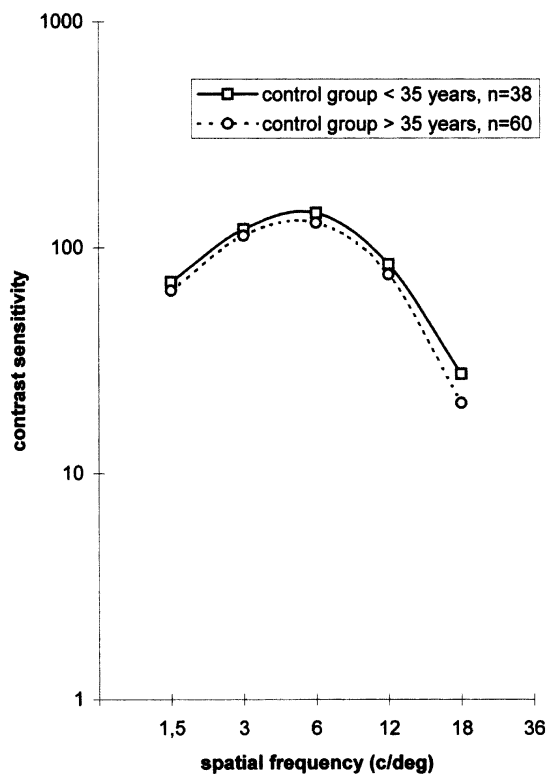

Fig. 1a: Contrast sensitivity curve in controls Comparison of CSFs in group of normals under 35 and over 35 years old

\begin{tabular}{|l|c|c|c|c|c|}
\hline Spatial frequency (c/deg) & 1.5 & 3 & 6 & 12 & 18 \\
\hline Parametr $\rho$ & 0.100 & 0.230 & 0.010 & 0.080 & 0.001 \\
\hline
\end{tabular}

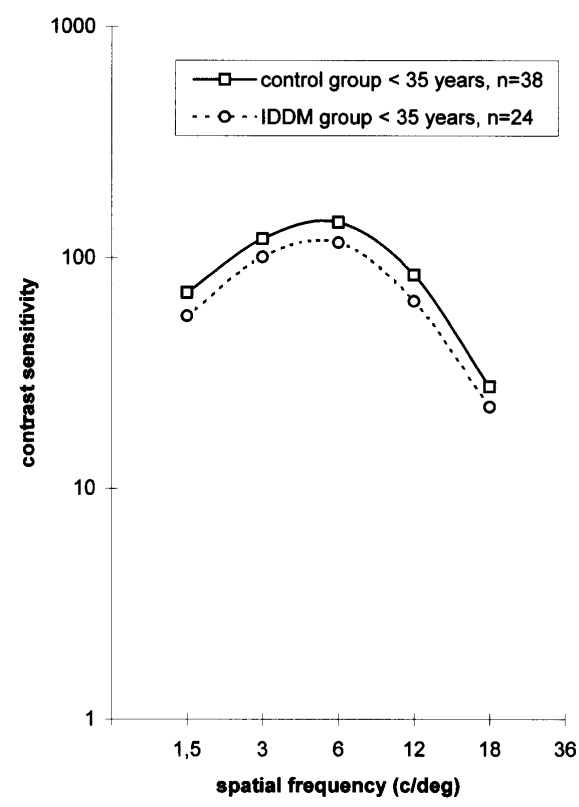

Fig. 2a: Contrast sensitivity curve in IDDM - age group under 35 years

Comparison with age matched control group

\begin{tabular}{|l|c|c|c|c|c|}
\hline Spatial frequency (c/deg) & 1.5 & 3 & 6 & 12 & 18 \\
\hline Parametr $\rho$ & 0.006 & 0.029 & 0.003 & 0.002 & 0.030 \\
\hline
\end{tabular}

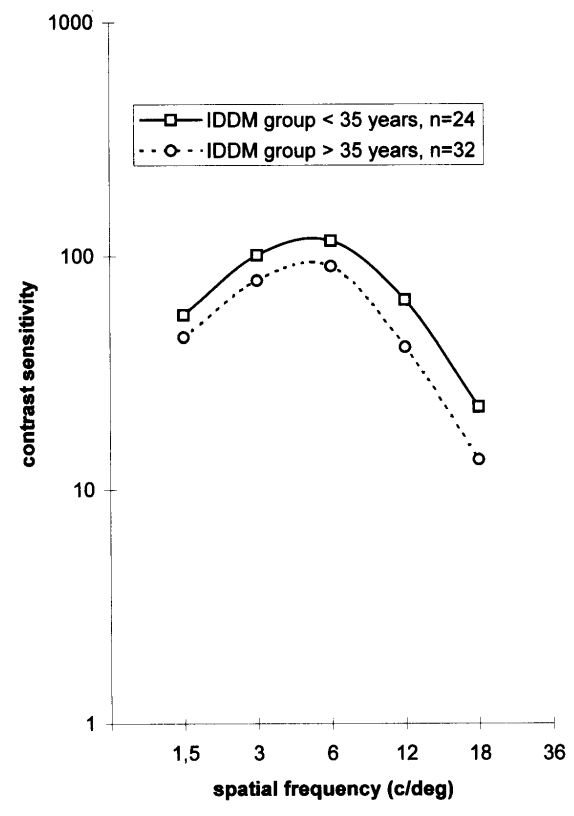

Fig. 1b: Contrast sensitivity curve in IDDM

Comparison of CSFs in group of diabetics under 35 and over 35 years old

\begin{tabular}{|l|c|c|c|c|c|}
\hline Spatial frequency (c/deg) & 1.5 & 3 & 6 & 12 & 18 \\
\hline Parametr $\rho$ & 0.026 & 0.007 & 0.007 & 0.001 & 0.001 \\
\hline
\end{tabular}

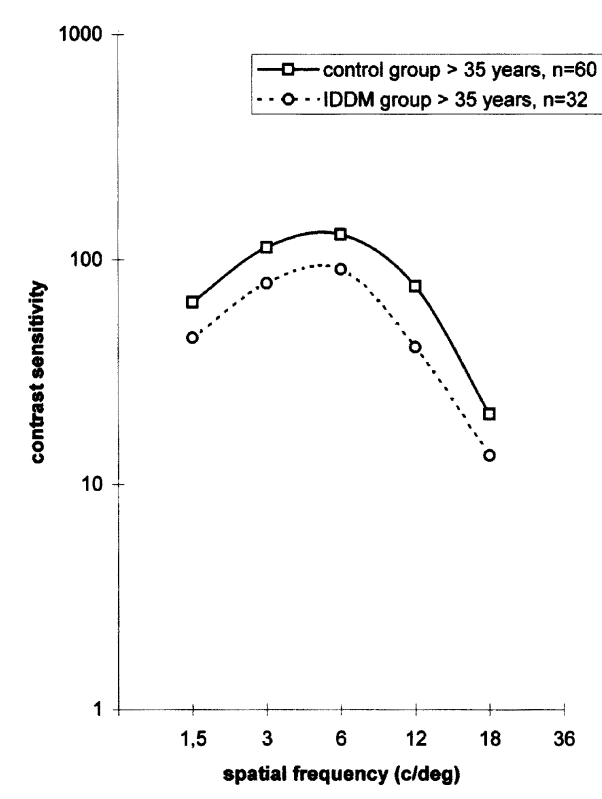

Fig. 2b: Contrast sensitivity curve in IDDM - age group over 35 years

Comparison with age matched control group

\begin{tabular}{|l|c|c|c|c|c|}
\hline Spatial frequency (c/deg) & 1.5 & 3 & 6 & 12 & 18 \\
\hline Parametr $\rho$ & 0.001 & 0.001 & 0.001 & 0.001 & 0.001 \\
\hline
\end{tabular}




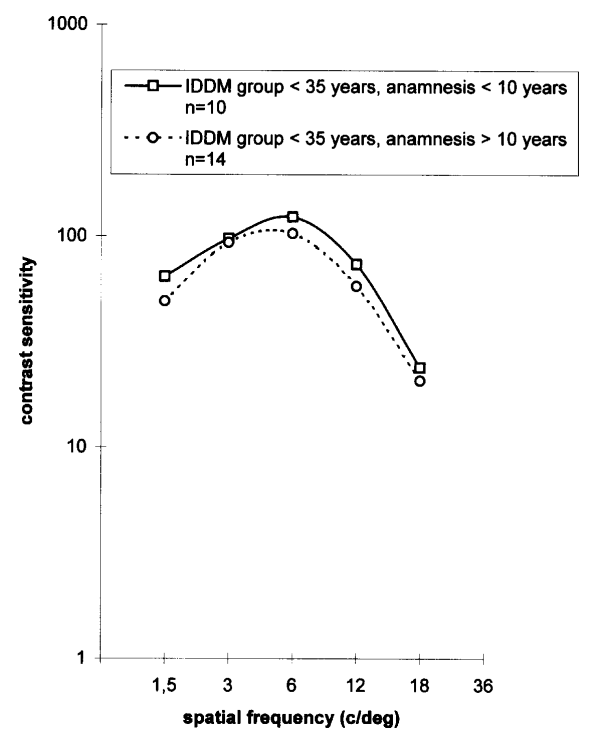

Fig. 3a: Influence of IDDM duration on CSFs - age group under 35 years

Comparison of the contrast sensitivity curves of diabetics being treated for less than 10 years and more than 10 years

\begin{tabular}{|l|c|c|c|c|c|}
\hline Spatial frequency (c/deg) & 1.5 & 3 & 6 & 12 & 18 \\
\hline Parametr $\rho$ & 0.050 & 0.350 & 0.036 & 0.040 & 0.227 \\
\hline
\end{tabular}

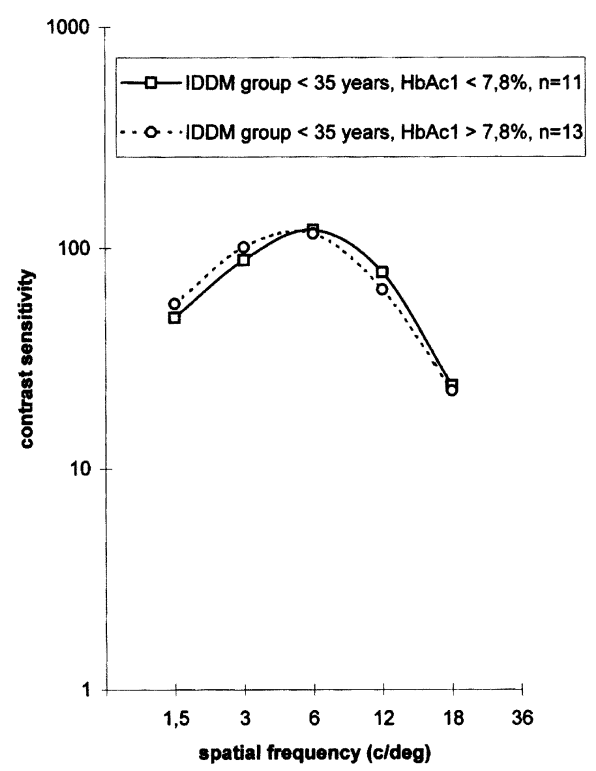

Fig. 4a: Influence of HbAlc value on CSFs - age group under 35 years

Comparison of the contrast sensitivity curves of diabetics with normal and pathological $\mathrm{HbA} 1 \mathrm{C}$ value

\begin{tabular}{|l|c|c|c|c|c|}
\hline Spatial frequency (c/deg) & 1.5 & 3 & 6 & 12 & 18 \\
\hline Parametr $\rho$ & 0.133 & 0.123 & 0.308 & 0.039 & 0.150 \\
\hline
\end{tabular}

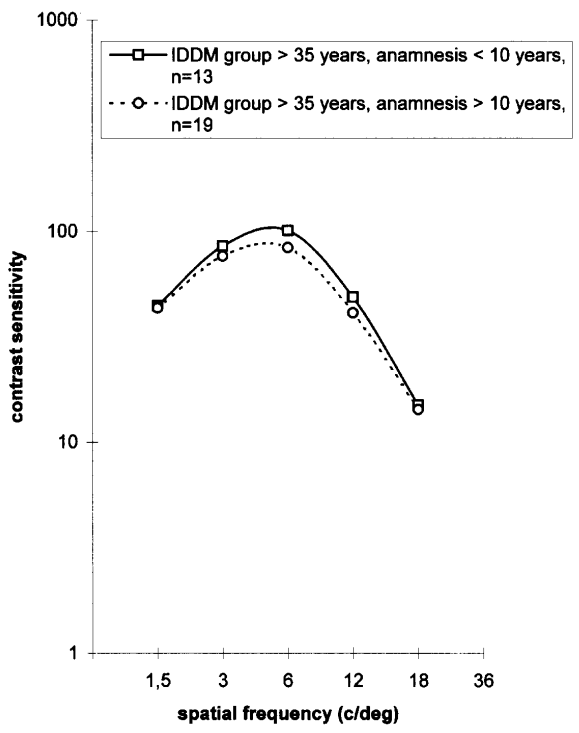

Fig. 3b: Influence of IDDM duration on CSFs - age group over 35 years

Comparison of the contrast sensitivity curves of diabetics being treated for less than 10 years and more than 10 years

\begin{tabular}{|l|c|c|c|c|c|}
\hline Spatial frequency (c/deg) & 1.5 & 3 & 6 & 12 & 18 \\
\hline Parametr $\rho$ & 0.460 & 0.085 & 0.007 & 0.140 & 0.350 \\
\hline
\end{tabular}

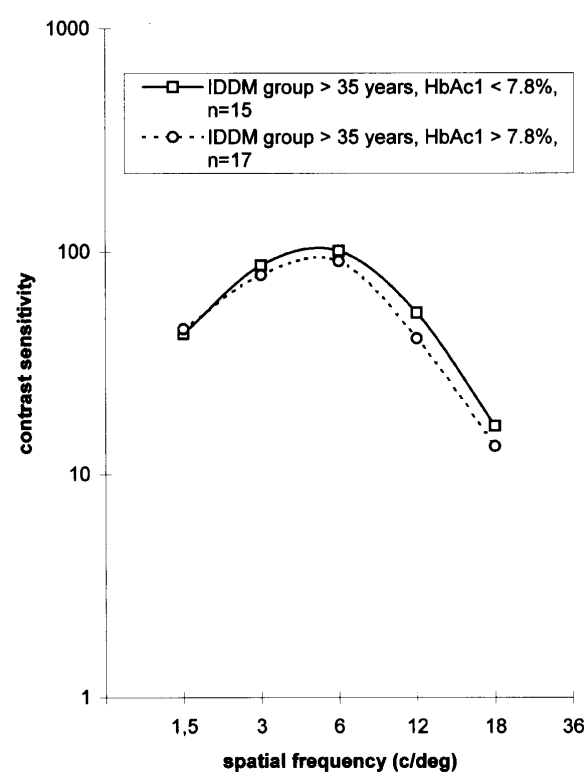

Fig. 4b: Influence of HbAlc value on CSFs - age group over 35 years

Comparison of the contrast sensitivity curves of diabetics with normal and pathological $\mathrm{HbA} 1 \mathrm{C}$ value

\begin{tabular}{|l|c|c|c|c|c|}
\hline Spatial frequency (c/deg) & 1.5 & 3 & 6 & 12 & 18 \\
\hline Parametr $\rho$ & 0.311 & 0.130 & 0.194 & 0.069 & 0.150 \\
\hline
\end{tabular}




\section{Discussion}

Contrast sensitivity is a complex and multilevel modality characterizing only a part of the human's visual function. The use of the VCTS 6500 (Vistech Consultants, Inc) is a valid and specific psychophysical test of the neurosensorial visual function's changes $(2,3,4,6,8)$.

Functions of contrast sensitivity are stable in healthy adult persons and show only a gradual and partial decrease during aging. The results of contrast sensitivity functions (CSFs) measurements for particular spatial frequencies at normals under 35 years were almost similar to the group comprised of normals over 35 years. Just the average values of CSFs in spatial frequency $6 \mathrm{c} / \mathrm{deg}$ and 18 $\mathrm{c} / \mathrm{deg}$ were significantly lowered in older persons (see Fig. 1a).

Our further results displayed a significant decrease in CSFs in all spatial frequencies in both IDDM groups (in the absence of any clinically recordable sign of the diabetic retinopathy), compared to the age and BCVA matched control groups (see Figs. 2a and 2b). In contradiction with similarities of the course of CSFs in both, age divided, control groups, the deterioration of CSFs in older IDDM patients was more profound in comparison with younger diabetics (see Fig. 1b).

Stationary grating CSF measurements obtained by Sokol et al. (9) via microprocessor controlled video system indicated that the paediatric IDDM patients without retinopathy were more sensitive than the normal subjects at extremly high and low spatial frequencies. This antagonistic finding should be an account of the difference in the mean age between the Sokol et al. group (31 patients, mean age 14.4 years) and our experimetal group A (19 patients, mean age $27,9 \pm 6.8$ ).

It is evident from our results that the biological age of a patient has a substantial influence on examined visual modality. Regarding the different findings in younger and older diabetics (and nearly identical findings in normal control groups) it is probable that the influence of the biological age of a patient is applicable as a mean of non-direct factor with a cumulative character, which potentiates destructive effect of IDDM.

Another factor which had a demonstrable influence on the extent of changes of CSFs at the IDDM was the duration of the disease. Decreased values of contrast sensitivity functions in diabetics being treated for 10 and more years if compared to findings at equally aged diabetics with shorter history of disease is considered to be a contributing discovery.

Statistically significant differencies in younger diabetics were found in the middle spatial frequencies of 6 and 12 c/deg (see Fig. 3a). Reduced influence of the disease's duration on CSFs in older diabetics (see Fig. 3b) is in contrast with the previous finding. Statistically significant change was proved only in spatial frequency $6 \mathrm{c} / \mathrm{deg}$. On the contrary, Mangouritsas et al. (7) did not record significant dif- ferences in the amount of CSFs reduction between two groups of IDDM divided by a time limit of 10 years of the disease's duration. The average age of patients in the Mangouritsas study was $32 \pm 8$ years.

Hypothetic age-related, non-direct (i.e. non-depending on IDDM), cumulating factor potentiating development of CSFs changes probably, besides extent, accelerated CSFs changes.

Our results (Figs. 4a and $4 \mathrm{~b}$ ) do not show any differences in contrast sensitivity at diabetics with normal levels of $\mathrm{HbA} 1 \mathrm{C}$ in comparison with groups with pathotogical results of examination of HbA1C. Just in the group of younger diabetics (< 35 years) there is barely statistically significant difference of CSFs on $12 \mathrm{c} / \mathrm{deg}$. A similar trend is described by Banford et al. (2) who refer to significant negative correlation of CSFs with $\mathrm{HbA} 1$ level at type I. diabetics in age 8-17 years (mean 13 years), as the HbAl increased the contrast sensitivity at 6 and $12 \mathrm{c} / \mathrm{deg}$ decreased. No relationship with actual blood glucose level were found during mentioned study. Some other studies are conflicting to this conclusions. According to Di-Leo et al. (5), the repetitive minor hypogylcaemic insults may contribute more to the neuronal damage than a marked or prolonged hyperglycaemic condition.

The pathophysiological substance of influencing the constrast sensitivity functions by diabetes is still not exactly known. The disturbance of visual functions may be linked to vascular damage and thus corelated to the degree of retinopathy. But this may not be a sole or primary case (2). Some speculations concerned the neurosensory dysfunction reflecting microvascular defects at a stage of no visible or minimal retinopathy (3). Arend et al. (1) concluded that the alteration of the perifoveolar capilary network in patients with diabetes are related to disturbance of the contrast sensitivity functions. Among others, Banford et al. (2) saw the possible reason also in changes in the retinal neurons metabolism or in the impairment of visual pathway on the higher levels. To clarify whether this is via tissue hypoxia related to the decreased oxygen uptake by neuroepithelium or due to direct toxic effect mediated by disturbed glucose-sorbitol pathway or reduction of essential fatty acid derivates is an challenge for further research.

Similarly as in another studies $(4,10)$ on CSFs in IDDM there were changes in spatial frequencies $6 \mathrm{c} / \mathrm{deg}$ and partially also $12 \mathrm{c} / \mathrm{deg}$ evaluated as the most sensitive ones among the investigated influences. This is in accordance with the fact that middle frequency targets require less contrast for detection (8).

\section{Conclusions}

We conclude that (1) age plays an important, suspectly non-direct, potentiating role on the patophysiology of the decreased CSFs in IDDM. (2) The partially different influence of diabetes duration on the drop of CSFs was proved in younger and older patients (3). 
Contrast sensitivity measurements can be used as a screening test completing Snellen BCVA for the detection of early visual impairment in IDDM. The most sensitive part of the test is the examination of CSFs by $6 \mathrm{c} / \mathrm{deg}$ and 12 $\mathrm{c} / \mathrm{deg}$. This issue has to be addressed further in a more extensive study.

\section{References}

1. Arend O, Remky A, Evans D, Stuber R, Harris A. Contrast sensitivity loss is coupled with capillary dropout in patients with diabetes. Invest Ophthalmol Vis Sci 1997;38(9):1819-24.

2. Banford D, North RV, Dolben J, Butler G, Owens DR. Longitudinal study of visual functions in young insulin dependent diabetics. Ophthal Physiol Opt 1994; 14:339-46.

3. Bangstad H-J, Brinchmann-Hansen O, Hultgren S, Dahl-Jorgensen K, Hanssen KF. Impaired contrast sensitivity in adolescents and young Type I (insulin-dependent) diabetic patients with microalbuminuria. Act Ophthalmol 1994;72:66873.

4. Brinchmann-Hansen O, Bangstad H-J, Hultgren S et al. Psychophysical visual function, retinopathy, and glycemic control in insulin-dependent diabetics with normal visual acuity. Act Ophthalmol 1993;71:230-57.

5. Di Leo MA, Capto S, Falsini B et al. Nonselective loss of contrast sensitivity in visual system testing in early type I. diabetes. Diabetes Care 1992;May,15(5):620-5.
6. Ginsburg AP. A new contrast sensitivity test chart. Am J Opthom Physiol 1984;61:403-7.

7. Mangouritsas G, Katouils E, Kepaptsoglou O, Zoupas C. Effekt von provozierter Hyperglykamie auf die Kontrastempfindlichkeitsfunktion bei insulinpflictigen Diabetikern. Ophthalmologe 1995;92(2):142-7.

8. Mannys MJ. Making sense of contrast sensitivity. Has its time come? Arch Ophthalmol 1987;105:627-9.

9. Sokol S, Moskowitz A, Skarf B, Evans R, Molitch M, Senior B. Contrast sensitivity in diabetics with and without background retinopathy. Arch Ophthalmol 1985;103:51-4.

10. Trick GL, Burde RM, Gordon MO, Santiago JV, Kilo C. The relationship between Hue discrimination and contrast sensitivity deficits in patients with diabetes mellitus. Ophthalmology 1988;95(5):693-8.

Submitted July 1999.

Accepted September 1999.

MUDr. Miroslav Dostálek, City Hospital, Department of Ophthalmology, J. E. Purkyně Str. 652, 57014 Litomyšl,

Czech Republic. e-mail: dostalek@lit.cz 